\title{
CIET-Analysis and Design of Folded Asymmetric H-Plane Waveguide Filters With Source-Load Coupling
}

\author{
Smain Amari and Jens Bornemann \\ Department of Electrical and Computer Engineering \\ University of Victoria, BOX 3055 \\ Victoria, B.C. V8W 3P6 \\ http://www.ece.uvic.ca
}

\begin{abstract}
The Coupled-Integral-Equations Technique (CIET) is used as a fast, accurate and reliable analysis tool for the design of folded asymmetric $H$-plane waveguide filters in which source-load coupling is used to add two finite transmission zeros. Designs examples of second-order filters with finite transmission zeros are presented. Numerical results are validated by comparison with results from the Finite Element Method (FEM) through the commercial software package HFSS.
\end{abstract}

\section{Introduction}

$\mathrm{C}$ YOUPLED resonator filters permeate modern $\checkmark$ communication systems where sharp cutoff skirts are required to effectively separate adjacent channels. In practice, these are often implemented using coupled resonators with additional couplings to generate finite transmission zeros [1-2].

The synthesis and design of coupled resonator filters have been the subject of intensive research efforts over the past 3 decades [1-3]. In the original model of $n$ coupled resonators proposed by Atia and Williams, it is assumed that the source feeds only resonator 1 while the load is coupled to only resonator $n$ [1]. It is well known that such a configuration can generate at most $n-2$ finite transmission zeros; this occurs when-among other couplings-resonators 1 and $n$ are cross coupled. Recently, configurations where the source or the load, or both, are coupled to more than one resonator and possibly to each other have been used to generate more than $n-2$ finite transmission zeros [4-6].

A simple realization of filters with source-load coupling consists in folded waveguide resonators with an additional coupling slot between the source and the load as shown in Figure 1. If the structure, such as that in Figure 2, is symmetric with respect to the center plane AA' at $a+t$, the analysis can be split into two stages corresponding to electric and metallic walls in this plane. The overall response of the filter is then obtained by adding and subtracting the individ- ual scattering coefficients, e.g., [7]. Unfortunately, in the stop-band of the filter, the scattering parameters of the two symmetries (even and odd) are comparable in magnitude; the difference between the two quantities can be accurately computed only when the scattering parameters of the two symmetry configurations are determined with even a higher accuracy. This issue is even more pronounced when finite transmission zeros are present in the stop band. Furthermore, the approach is evidently limited to symmetric structures.

To overcome these limitations, we avoid splitting the structure into its symmetries even when the structure is physically symmetric. A given discontinuity is viewed either as a slot or a bifurcation depending on whether it crosses the plane AA' or not. The entire filter is then analyzed in one step using the Coupled-Integral-Equations Technique (CIET) where basis functions with the proper edge-conditions are used [8]. The design is completed by an optimization procedure which utilizes the CIET as an accurate and reliable analysis tool.

\section{Theory}

We focus attention on Figure 1 which shows an asymmetric structure divided into different subregions. Following the Coupled-Integral-Equations Technique (CIET), coupled integral equations for the electric field at the discontinuities are derived and then solved by the moment method. Two different configurations must be considered: first, the bifurcation and, secondly, the asymmetric iris. Let $X_{1}(x)$, $X_{2}(x) \ldots$, and $X_{p}(x)$ denote the aperture electric fields ordered from source to load as shown in Figure 1. Starting from modal expansions of the electric and magnetic fields in the different regions of the structure, and enforcing the continuity of the magnetic field at apertures 1,2 and 3, we get the following integral equations: 


$$
\begin{array}{r}
\sum_{n=1}^{\infty} j Y_{n}^{I} \tilde{X}_{1}^{I}(n) \phi_{n}^{I}(x) \\
+\sum_{n=1}^{\infty} Y_{n}^{I I} \frac{\tilde{X}_{1}^{I I}(n)+\tilde{X}_{p}^{I I}(n)}{\tan \left(k_{z n}^{I I} d_{1}\right)} \phi_{n}^{I I}(x) \\
-\sum_{n=1}^{\infty} Y_{n}^{I I} \frac{\tilde{X}_{2}^{I I}(n)+\tilde{X}_{p-1}^{1}(n)}{\sin \left(k_{z n}^{I I} d_{1}\right)} \phi_{n}^{I I}(x) \\
=-2 j Y_{1}^{I} \phi_{1}^{I}(x), \quad 0 \leq x \leq a \\
\sum_{n=1}^{\infty} Y_{n}^{I I} \frac{\tilde{X}_{2}^{I I}(n)+\tilde{X}_{p-1}^{I I}(n)}{\tan \left(k_{z n}^{I I} d_{1}\right)} \phi_{n}^{I I}(x) \\
-\sum_{n=1}^{\infty} Y_{n}^{I I} \frac{\tilde{X}_{1}^{I I}(n)+\tilde{X}_{p}^{I I}(n)}{\sin \left(k_{z n}^{I I} d_{1}\right)} \phi_{n}^{I I}(x) \\
+\sum_{n=1}^{\infty} Y_{n}^{I I I} \frac{\tilde{X}_{2}^{I I I}(n)}{\tan \left(k_{z n}^{I I I} d_{2}\right)} \phi_{n}^{I I I}(x) \\
-\sum_{n=1}^{\infty} Y_{n}^{I I I} \frac{\tilde{X}_{3}^{I I I}(n)}{\sin \left(k_{z n}^{I I I} d_{2}\right)} \phi_{n}^{I I I}(x)=0
\end{array}
$$

and

$$
\begin{array}{r}
\sum_{n=1}^{\infty} \frac{Y_{n}^{I I I}}{\tan \left(k_{z n}^{I I I} d_{2}\right)} \tilde{X}_{3}^{I I I}(n) \phi_{n}^{I I}(n) \\
\sum_{n=1}^{\infty} \frac{Y_{n}^{I V}}{\tan \left(k_{z n}^{I V} d_{3}\right)} \tilde{X}_{3}^{I V}(n) \phi_{n}^{I V}(n) \\
-\sum_{n=1}^{\infty} \frac{Y_{n}^{I I I}}{\sin \left(k_{z n}^{I I I} d_{2}\right)} \tilde{X}_{2}^{I I}(n) \phi_{n}^{I I I}(x) \\
-\sum_{n=1}^{\infty} \frac{Y_{n}^{I V}}{\sin \left(k_{z n}^{I V} d_{3}\right)} \tilde{X}_{4}^{I V}(n) \phi_{n}^{I V}(x)=0 .
\end{array}
$$

Here, $Y_{n}^{i}$ and $k_{z n}^{i}$ are the wave admittances and the propagation constants of the $\mathrm{TE}_{n 0}$ mode in the $\mathrm{i}^{\text {th }} \mathrm{sec}-$ tion. Equation (2) holds over the aperture between regions II and III whereas equation (3) is valid over the aperture between regions III and IV. The transform $\tilde{X}_{m}^{i}(n)$ is defined by

$$
\tilde{X}_{m}^{i}(n)=\int X_{m}^{i}(x) \phi_{n}^{i}(x) d x
$$

where the integral extends over the range of $X_{m}(x)$ and $\phi_{n}^{i}(x)$ is the normalized modal distribution of the magnetic field of the $\mathrm{TE}_{n 0}$ mode in the $\mathrm{i}^{\mathrm{th}}$ section, e.g., $\phi_{n}^{I}(x)=\sqrt{\frac{2}{a}} \sin \left(\frac{n \pi x}{a}\right)$. The remaining integral equation are similar to one of equations (1), (2) or (3) and are therefore omitted. The derivation of these equations follows straightforwardly from the discussion in [8].

To guarantee the efficiency of the numerical solution, edge-conditioned basis functions are constructed from the modes of the individual subregions by introducing appropriate weighting factors which include the proper field singularities at the discontinuities. The application of the moment method, in the form of Galerkin's method, requires the evaluation of integrals involving products of the basis functions and the modes of the individual sections; these can be expressed in terms of Bessel functions of order $1 / 6$ [8]. The resulting matrix equation in the expansion coefficients has several distinct features [9]. It is extremely sparse with a known block structure which is exploited through a customized inversion routine based on LU decomposition.

Once the coupled integral equations are solved, the scattering parameters of the modes of interest at the two ports follow straightforwardly, e.g. [9]. Note that no distinction is made between symmetric and asymmetric structures.

The design is carried out by calculating a modified inter-cavity coupling matrix, which includes the source-load coupling, from given filter specifications. Initial dimensions are determined from the respective CIET analysis routines by varying a single dimensional parameter for every entry of the modified coupling matrix. These parameters are subsequently used in the fast and reliable CIET analysis of the overall filter where an optimization algorithm varies a given set of dimensions until the filter specifications are met.

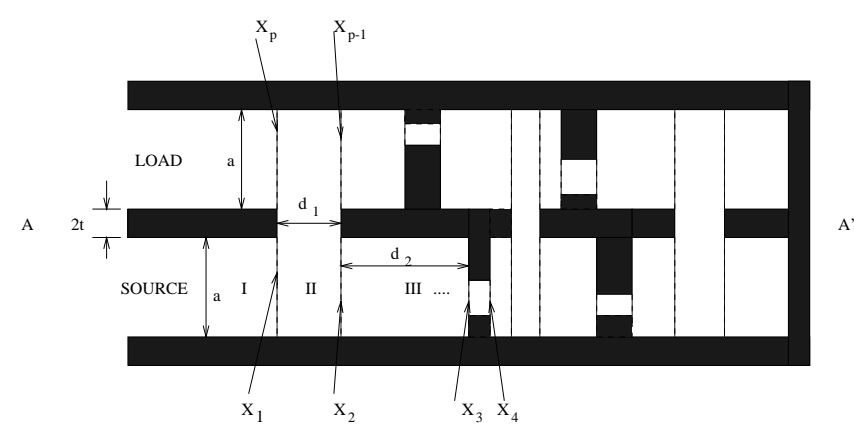

Fig. 1. Typical top-view of a folded H-plane waveguide resonator filter with source-load coupling.

\section{Results}

A number of filters with different requirements were analyzed and optimized using the procedure described above.

Two two-resonator filters which give the same magnitude response but which are physical different were 


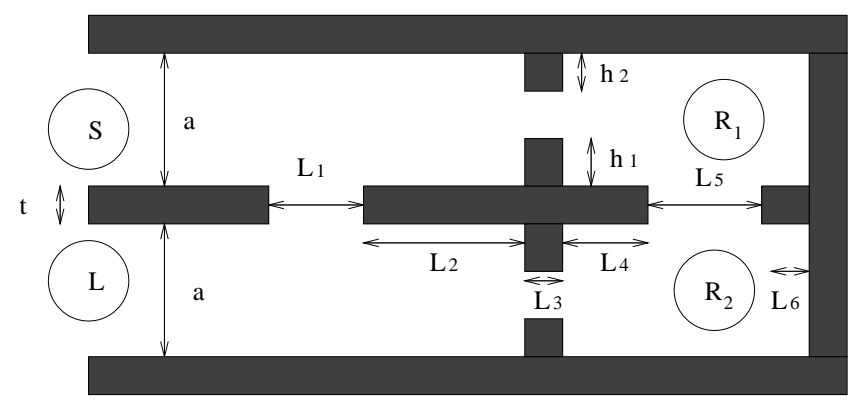

Fig. 2. H-plane structure used to implement Filters 1 and 3.

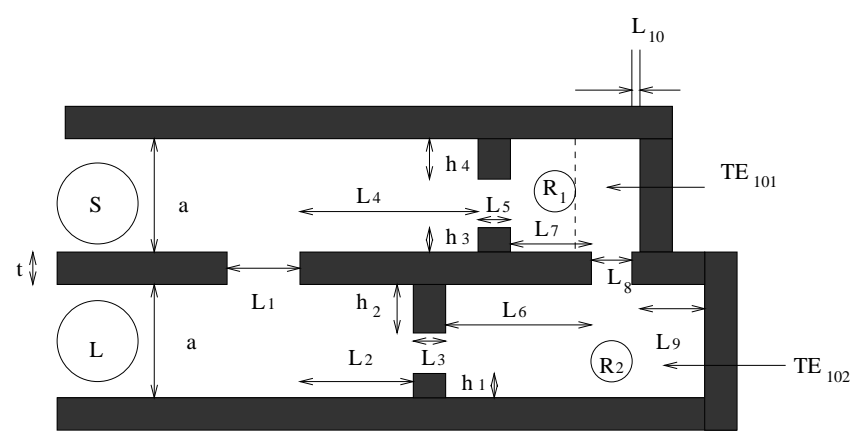

Fig. 3. H-plane structure used to implement Filters 2 and 4.

designed to satisfy the following specifications. The in-band return loss is $23 \mathrm{~dB}$ and the normalized transmission zeros are located at $j \omega= \pm j 8$. The insertion and return loss of the two filters are shown in Figure 4. The solid line represents the response of Filter 1 whose layout is shown in Figure 2 and whose dimensions (in mm) are: $a=19.05, L_{1}=20.560, L_{2}$ $=5.053, L_{3}=4.066, L_{4}=2.094, L_{5}=12.140, L_{6}$ $=0.318, h_{1}=3.503, h_{2}=5.783$, and $t=4.501 \mathrm{~mm}$. The dashed line in Figure 4 shows the response of the Filter 2 where the source-load coupling coefficient is negative. The layout of this filter is shown in Figure 3 and its dimensions (in $\mathrm{mm}$ ) are: $a=19.05, \mathrm{t}=1.00$, $L_{1}=5.458, L_{2}=0.741, L_{3}=0.320, L_{4}=7.634, L_{5}$ $=7.990, L_{6}=16.409, L_{7}=9.037, L_{8}=7.132, L_{9}$ $=9.993, L_{1} 0=0.020, h_{1}=3.407, h_{2}=7.897, h_{3}=$ $1.709, h_{4}=9.176 \mathrm{~mm}$. The height of the main waveguide is $9.525 \mathrm{~mm}$ in both filters.

Up to 4 basis functions with the proper edge conditions and up to 50 terms were summed in computing the inner products in both cases. More basis functions and terms were also used and led to insignificant changes. The next example is presented to show the performance of narrow-band filters which implement using the symmetric structure in Figure 2. The stopband of these filters is free from spurious bands and maintains a high level of attenuation as shown in Figure 5. Indeed, with only two resonators a minimum insertion loss of over $60 \mathrm{~dB}$ is maintained over the stopband. The two finite transmission zeros which are brought about by the source-load coupling allow

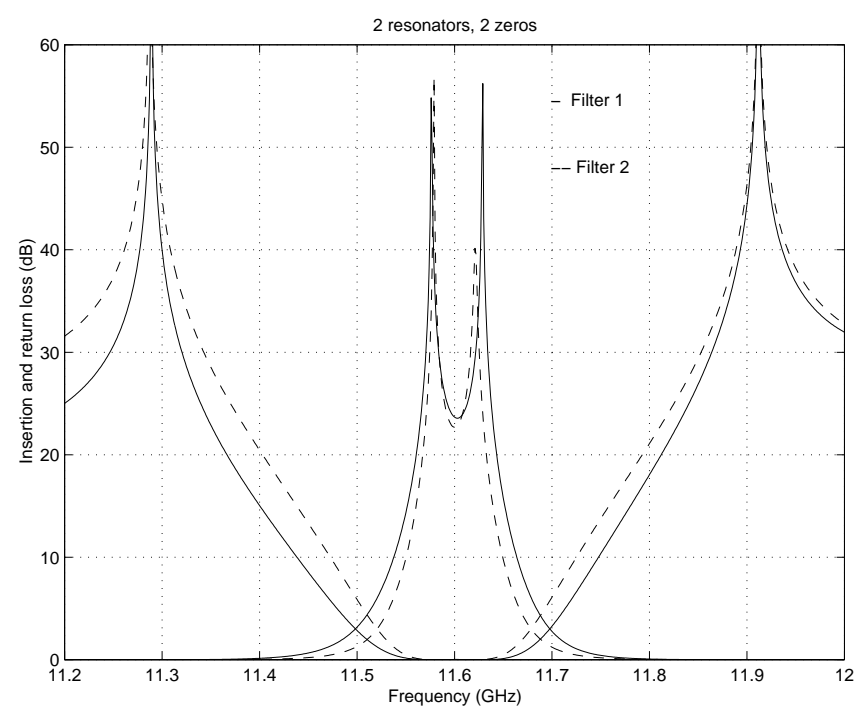

Fig. 4. Insertion and return loss of Filters 1 and 2. The dimensions of the filters are given in the text.

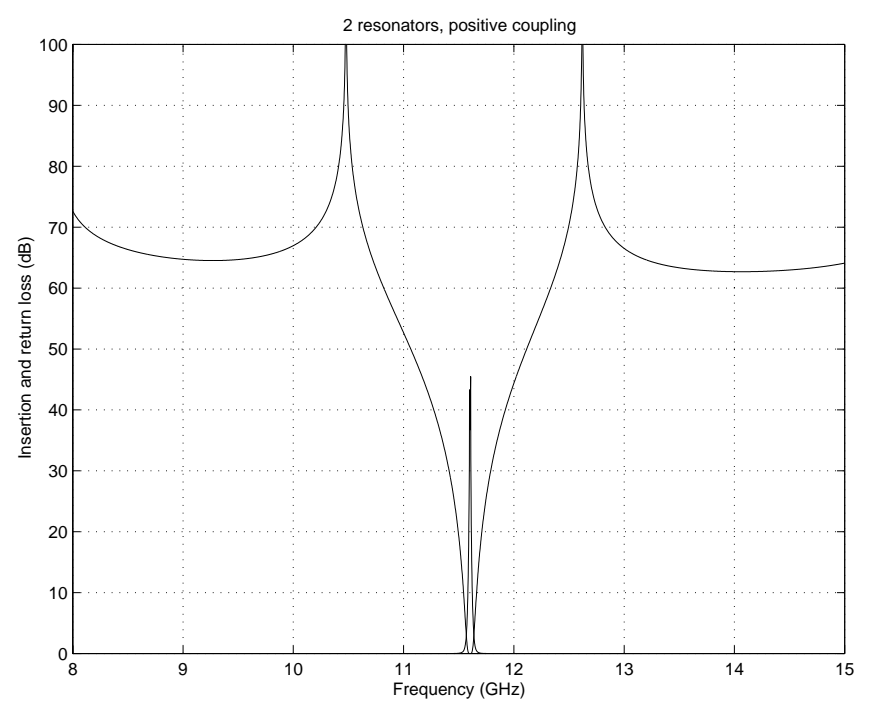

Fig. 5. Insertion and return loss of Filter 3.

a sharp cutoff. Without the source-load coupling the zeros of a second order filter are both located at infinity (assuming frequency independent coupling coefficients). The present example clearly demonstrates the potential advantage of the source-load coupling despite the fact that the isolation of this type of filters is always finite since it has the same number of poles and zeros. The filter (Filter 3 ) shown in Figure 5 was designed using the CIET with 4 basis functions and 50 terms in the sums. Its dimensions are (in $\mathrm{mm}$ ): $a=19.05, L_{1}=0.618, L_{2}=5.596, L_{3}=2.187, L_{4}$ $=1.406, L_{5}=3.724, L_{6}=11.465, h_{1}=5.957, h_{2}=$ 6.289 , and $t=1 \mathrm{~mm}$. The height of the main waveguide is $9.525 \mathrm{~mm}$. An expansion of the passband of this filter is shown in Figure 6 where the two reflection zeros are clearly visible.

In order to validate the computer code used in 


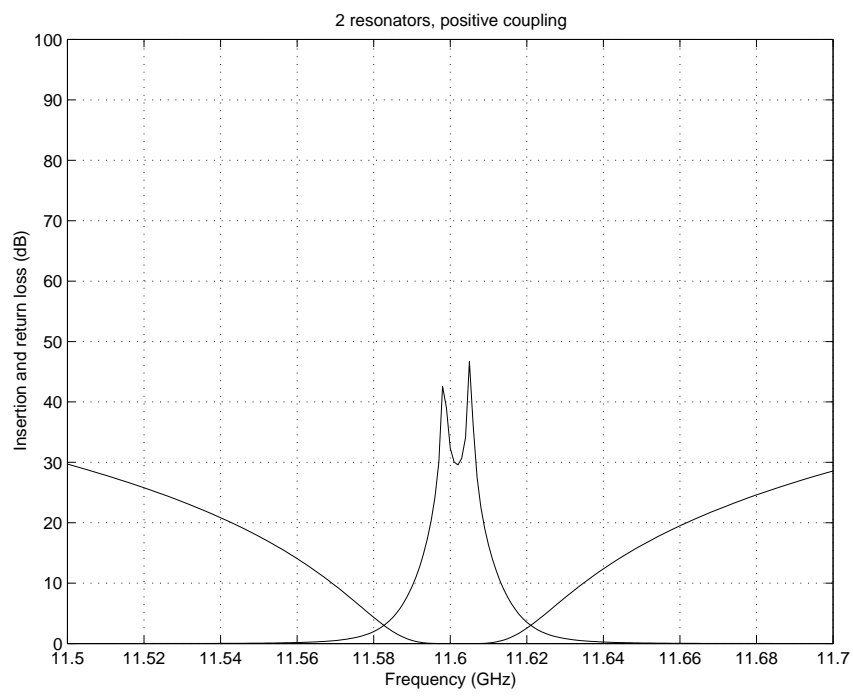

Fig. 6. Passband of filter III.

the design of these filters, a filter (Filter 4) was designed using the same approach and then analyzed using both the CIET and the Finite Element Method through the software package HFSS. The insertion and return loss of the designed filter as a function of frequency is are shown in Figure 7. The solid line is obtained from HFSS and the dashed line is from the CIET using 4 basis functions. The agreement between the two methods is good. The dimensions (in $\mathrm{mm}$ ) of the filter which is implemented using the structure in Figure 3 are : $a=19.05, \mathrm{t}=1.00, L_{1}$ $=5.458, L_{2}=1.926, L_{3}=0.339, L_{4}=7.769, L_{5}=$ $0.829, L_{6}=16.494, L_{7}=10.16, L_{8}=6.374, L_{9}=$ $11.059, L_{1} 0=0.015, h_{1}=7.226, h_{2}=5.117, h_{3}=$ $2.177, h_{4}=9.53 \mathrm{~mm}$. The height of the main waveguide is $9.525 \mathrm{~mm}$.

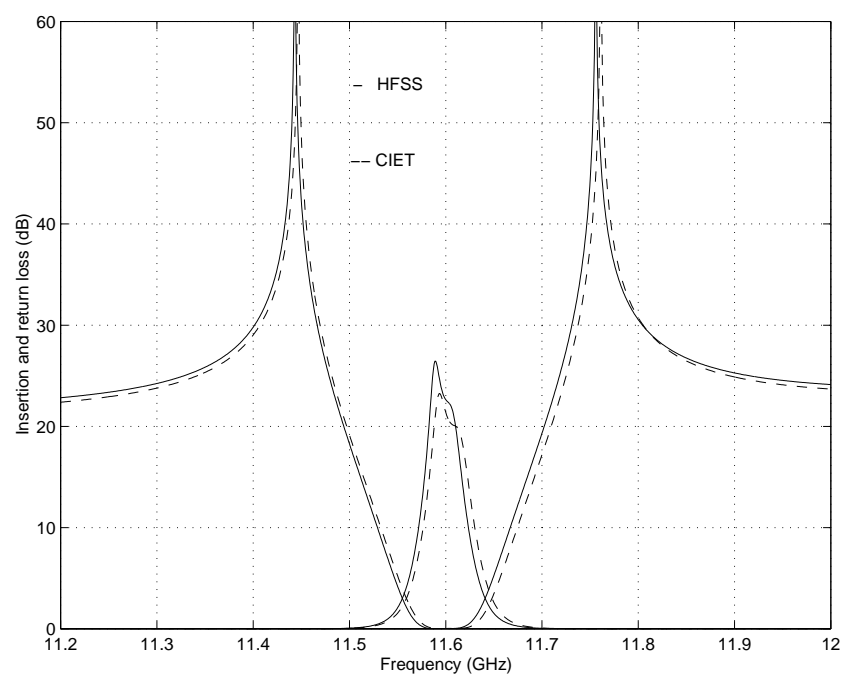

Fig. 7. Insertion and return loss of Filter 4. The solid line is from the Finite Element Method (HFSS) and dashed line from CIET.

\section{Conclusions}

Folded H-plane resonator waveguide filters with source-load coupling are designed and analyzed using the Coupled-Integral-Equation Technique (CIET). The additional coupling of the source and the load is used to add two finite transmission zeros and enhance the stop band of the filters. Numerical results were validated by comparison with the Finite Element Method (FEM) through the commercial software package HFSS.

\section{V. acknowledgment}

The authors would like to thank Uwe Rosenberg of Bosch Telecom (now Marconi Communications), Backnang, Germany for helpful discussions and Alexandre Laisné for the HFSS simulations.

\section{REFERENCES}

[1] A. Atia and Williams, "New type of waveguide bandpass filters for satellite transponders", COMSAT Tech. Rev., vol. 1, No. 1, pp. 21-43, 1971.

[2] R. J. Cameron, "General coupling matrix synthesis methods for Chebychev filtering functions", IEEE Trans. Microwave Theory Techn., vol. 47, pp. 433-442, Apr. 1999.

[3] Special issue, IEEE Transactions Microwave Theory Tech., Sept. 1982.

[4] J. F. Liang and W. D. Blair, "High-Q TE01 mode DR filters for PCS wireless base stations", IEEE Trans. Microwave Theory Techn., vol. 46, pp. 2493-2500, Dec. 1998.

[5] K. A. Zaki, C. Chen and A. Atia, "Modeling of coupling probes in dual mode cavities", IEEE MTT-S Int. Symp. Dig., New York 1988, pp. 515-518

[6] U. Rosenberg and W. Hägele, "Advanced multimode cavity filter design using source/load-resonance circuit cross couplings", IEEE Microwave Guided Wave Lett., vol. 2, pp.508-510, Dec. 1992.

[7] G.L. Matthaei, L. Young and E.M.T. Jones, Microwave Filters, Impedance Matching Networks, and Coupling Structures, Artech House, Boston, 1980.

[8] S. Amari, J. Bornemann and R. Vahldieck, "Accurate analysis of scattering from multiple waveguide discontinuities using the coupled-integral-equation technique”, J. Electromag. Waves Appl., vol. 10, pp.1623-1644, Dec. 1996.

[9] J. Bornemann, S. Amari and R. Vahldieck, "Analysis of waveguide discontinuities by the coupled-integral-equations technique", $R e$ cent Res. Devel. Microwave Theory \& Tech., Vol. 1, pp. 25-35 Dec. 1999. 